\title{
Past plant diversity changes and future conservation issues
}

\author{
Rachid Cheddadi' , S. Báez² , S. Normand ${ }^{3}$, D. Payne ${ }^{4}$ and P. Taberlet ${ }^{5,6}$
}

Past climate changes have shaped the distribution of species across the planet by impacting their dispersal abilities, their migration rates, and the ongoing competition between and among them, as well as by trig gering their local or total extinctions. This, in turn, has led to major changes in community composition and to ecosystem turnovers.

Ongoing climate change is affecting species as well, and will keep doing so in the future. However, the challenge that we face today is that climate change is happening much faster than in the past and exceeding the migration rates of many species, driving a need for informed management to support the natural ability of species to respond. Moreover, climate change is no longer the only important factor in determining biodiversity patterns.

Changes in diversity are driven by complex interactions between climate change and increasing human pressures, whether from large-scale political and socio-economic transformations, overexploitation and unsus tainable management of natural resources, or demographics. The accelerated growth of the human population, for example, is likely to dramatically reduce and fragment the range of species' natural habitats, thereby threatening their potential for long-term persistence. In this modern context, many plant species will have to adapt locally or will become extinct because their natural dispersal to more suitable habitats will be compromised.

The evolutionary history of plants and their communities and of their response to environmental drivers started long ago and has followed various pathways. Accordingly, there are as many explanations for current patterns of diversity as there are species, and each species has a unique history among its coevals. Over geological time, certain plant species have adapted locally or migrated to more suitable habitats while others are now extinct because of their low dispersal abilities and/or reduced capacities for adaptation to new environments (e.g. the Atlas cedar in the Rif Mountains in Morocco; Fig. 1). For example, during the last glacial period, many temperate plant species persisted with low densities and/or with restricted ranges in so-called "refugia", many of which are found in mountains. Phylogeographical studies have shown that many of these glacial refugia are hotspots of genetic and species diversity that result from a high relative abundance of endemic and ancestral genes and marked genetic differentiation at the local scale.

Relicts of the past can provide us with fascinating and useful information on how species reacted to different climatic situations, which in turn could inform management studies combining surveys of modern adaptation to environmental changes. climate changes and biodiversity. strategies to protect the remaining species on Earth now and in the future. For example, fossil records contain a wide range of proxies for reconstructing past climates, species occurrences, their range of fluctuations, and their extinction through time. Accordingly, DNA and fossil records allow us to trace Quaternary population and range dynamics with unprecedented detail, while studies on ancient DNA extracted from geological records are providing new insights into species

Models offer another way to inform management strategies and species' conservation policies. Modeling the ancient distribution of plant species illustrates potential migration pathways and refugia, and can increase our understanding of the demographic history and current genetic structure of species. However, numerous decisions and challenges accompany such modeling efforts, including the choice of modeling paradigm and algorithm, biases in algorithms and distribution data, variability in estimates of paleoclimate, means of model validation, genetic heterogeneity and model equilibrium, estimates of dispersal capacities, and our understanding of the lability of species' environmental tolerance and biotic interactions.

Management strategies and species' conservation policies can certainly be optimized through the integration of knowledge from different sources, disciplines, and times cales. However, the implementation of such strategies requires multi-scale, multi-species, and multi-disciplinary approaches to evaluate the relationship between long-term

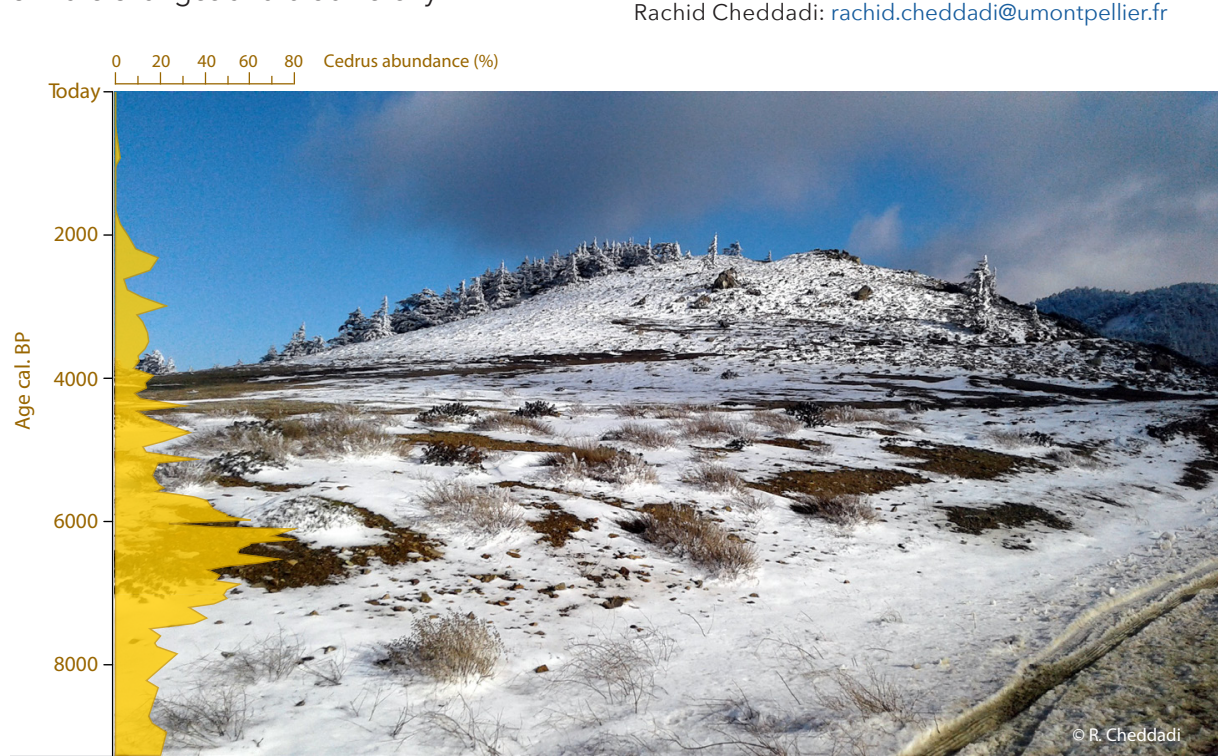

Figure 1: The fossil record shows that Atlas cedar (Cedrus atlantica), which currently is endangered, formerly comprised significantly more extended forests in the Rif Mountains (Morocco). This picture shows a relict population of the Atlas cedar at $\sim 1800 \mathrm{~m}$ above sea level in the Rif Mountains. Snow still occurs in winter but the increase of winter temperature and the steady decrease of annual precipitation over the past few decades is threatening the local persistence of the Atlas cedar in North Africa.

In this issue we present highlights from research on past climate changes, genetic approaches, and vegetation modeling to examine the relationship between past environmental changes and their impacts on different aspects of plant species' diversity. We then discuss how such approaches could inform conservation and management.

The articles in this issue fall into four main sections: (1) Issues related to past climate variability in terms of amplitude, velocity, and abruptness, and how they have affected where species have persisted during climatically unsuitable time periods (Braconnot and Vimeux p. 4; Zheng et al. p. 6; Cheddadi and Bennett p. 8), (2) Impacts of long-term isolation and migration of species on their genetic diversity (Ficetola and Taberlet p. 10 Alsos et al. p. 12; Parducci p. 14), (3) Models of past species' distributions and biases to modeling approaches (François and Hambuckers p. 16), and finally (4) Lessons from the past to inform plant species conservation in the future (Payne et al. p. 18; Dirk and Gillson p. 20; Sarmiento et al. p. 22; Castilla-Beltrán et al. p. 24).

\section{AFFILIATIONS}

IISEM, University of Montpellier, CNRS, IRD, France Departamento de Biologia, Escuela Politécnica Nacional del Ecuador, Quito, Ecuador ${ }^{3}$ Arctic Research Centre, Aarhus University, Denmark "Global Mountain Biodiversity Assessment (GMBA), Institute of Plant Sciences, University of Bern Switzerland

'Laboratoire d'Ecologie Alpine (LECA), CNRS Université Grenoble Alpes, France

${ }^{6}$ The Arctic University of Norway (UiT), Troms $\varnothing$ Museum, Norway

\section{CONTACT}

